\title{
The phonological loop model of working memory: An ERP study of irrelevant speech and phonological similarity effects
}

\author{
MANUEL MARTÍN-LOECHES \\ Complutense University, Madrid, Spain \\ STEFAN R. SCHWEINBERGER \\ University of Constance, Constance, Germany \\ and \\ WERNER SOMMER \\ Humboldt University, Bertin, Germany
}

\begin{abstract}
The phonological loop model for retention of auditory verbal material in working memory, developed by Baddeley, assumes that irrelevant speech and phonological similarity influence only one and the same element of the system - that is, the phonological short-term store. We tested this idea by recording eventrelated potentials (ERPs) to auditorily presented letters that were phonologically similar or dissimilar and were to be memorized in the presence of more or less disturbing irrelevant speech. Irrelevant speech and phonological similarity caused ERP effects with clearly different scalp topographies, indicating that these factors influence different brain systems and hence probably different cognitive elements. Moreover, ERPs indicated that the phonological similarity effect might involve processes at the level of phonological analysis. Our data also support recent suggestions that the irrelevant speech effect is not based on the phonological similarity between relevant and irrelevant material, but on the phonological variability within the irrelevant stream.
\end{abstract}

Baddeley's model of working memory proposes the existence of an attentional control system (the central executive) and two slave systems. These slave systems are the visuospatial sketch pad for visual images and the phonological loop for speech-based information (Baddeley, 1986, 1990, 1992b, 1992c). The phonological loop is assumed to be composed of two subsystems or elements: a phonological short-term store to hold acoustic or speech-based information, and an articulatory control process, analogous to inner speech, used to maintain material within the phonological store. The articulatory control process is also used to transfer visually presented verbal material into the phonological store by subvocalization, whereas auditorily presented verbal material gains direct and obligatory access to the phonological shortterm store. Evidence for this description of the phonological loop has come from many studies (e.g., Baddeley, 1986; Belleville, Peretz, \& Arguin, 1992; Vallar \& Cappa, 1987; Vallar, Corno, \& Basso, 1992).

We thank Eva-Maria Pfütze for her help in data collection. This research was supported by a grant of the Ministerio de Educación y Ciencia (Spain) to M.M.-L. and by the Deutsche Forschungsgemeinschaft (So 177/4-2). Correspondence should be addressed to M. MartínLoeches, Brain Mapping Unit, Pluridisciplinar Institute, Complutense University, Po. Juan XXIII, 1. E-28040-Madrid, Spain (e-mail: mmartin@ eucmax. sim.ucm.es)
The theoretical conceptions about the phonological short-term store are based to a large extent on the consequences of two experimental manipulations on recall performance, the "irrelevant speech effect" and the "phonological similarity effect." The irrelevant (or unattended) speech effect is the impairment of recall when irrelevant auditory material is presented together with the items to be memorized. It is explained by the obligatory access of the irrelevant material to the phonological short-term store, where it is supposed to interfere with representations of the memorized items (Baddeley, 1986, 1990, 1992b; Gathercole \& Baddeley, 1993; Jones \& Morris, 1992). There are two models regarding the mechanism of the irrelevant speech effect. Baddeley conceives the effect to be due to the phonological similarity between the relevant and the irrelevant material; the higher this similarity, the stronger the effect. This is supported by findings that white noise and material that is phonologically different from the relevant items do not produce the irrelevant speech effect, and nonvocal music is less disruptive than vocal music, which in turn is less disruptive than speech (Hanley \& Broadbent, 1987; Salamé \& Baddeley, 1982, 1986, 1989). In contrast, Jones and co-workers have recently suggested that the irrelevant speech effect is due to the changes in sound in the irrelevant stream from one utterance to the next. The higher the phonological or acoustical variability within the irrelevant material, the stronger this effect. In support of this idea, it has been reported that even non- 
phonological material such as pure tones may cause the irrelevant speech effect, provided they are variable in pitch (Jones, 1994; Jones \& Macken, 1993, 1995; Jones, Madden, \& Miles, 1992; Jones \& Morris, 1992).

The phonological similarity effect is the impairment of recall performance when the memorized items are phonologically similar to one another (e.g., the letters $B$, $D, P$ ) as compared to when they are dissimilar (e.g., $J, Q$, $X$; Baddeley, 1966; Longoni, Richardson, \& Aiello, 1993; Papagno \& Vallar, 1992; Salamé \& Baddeley, 1982, 1986; Vallar \& Cappa, 1987). The phonological similarity effect is explained by the principle that losing a phonological feature that discriminates one item from the others in the phonological short-term store is more detrimental when there are fewer discriminating features (Baddeley, 1986, 1990; Gathercole \& Baddeley, 1993).

Irrelevant speech and phonological similarity effects are considered to influence only one and the same element of the phonological loop-that is, the phonological short-term store (Baddeley, 1986, 1990, 1992b; Baddeley \& Salamé, 1986; Miles, Jones, \& Madden, 1991). Therefore, one should expect an interaction of these effects when both are present together (Baddeley, 1992a). However, neither Salamé and Baddeley (1986) nor Jones and Macken (1995) found such an interaction to be statistically significant, although the numerical effects of irrelevant speech were relatively small under phonological similarity conditions. Baddeley (1992a) considers this absence of an interaction between the two effects as a major problem of his model. Although this does not necessarily mean that both effects influence different elements, the assumption that both effects have the same locus of action should be further explored.

One approach to elucidate whether irrelevant speech and phonological similarity affect one and the same element of working memory (the phonological short-term store) could be the use of event-related brain potentials (ERPs), which consist of brain electrical activity timelocked to the occurrence of an event. The effect of an experimental manipulation on the ERP can be directly measured as a "difference wave"- that is, subtracting the ERP obtained under a condition in which the experimental effect in question is absent or small from the ERP recorded when it is present. With this procedure, the activity of elements equally involved in both conditions is eliminated, leaving only the activity linked to the elements specifically affected by the experimental manipulation. The topography of ERPs across the scalp reflects the location and orientation of the generating brain systems. Although it is difficult to anatomically localize the underlying brain systems, it is possible to infer the involvement of different generating brain systems from different ERP topographies (McCarthy \& Wood, 1985). The topography of the components observed in the difference waves can thus be compared in order to assess whether they reflect the activity of similar or different brain sources. Because it is unlikely that one and the same cognitive element is located in different neural substrates, cognitive elements may also be dissociated on the basis of different scalp topographies (see, e.g., Johnson, 1993). If ERP difference waves obtained under irrelevant speech and phonological similarity conditions show unequal topographies, it is likely that these effects modulate the activity of different brain loci and, hence, probably relate to different loci of action at the cognitive level.

Although numerous ERP studies have investigated verbal short-term memory (e.g., Lang, Starr, Lang, Lindinger, \& Deecke, 1992; Ruchkin, Johnson, Grafman, Canoune, \& Ritter, 1992; for a review, see Starr, Barrett, Pratt, Michalewski, \& Patterson, 1990), irrelevant speech or phonological similarity have never been manipulated. Therefore, no conclusion can be drawn from these studies about the question of a common locus of action of these variables.

The main aim of the present study was to investigate with the ERP technique whether or not irrelevant speech and phonological similarity effects modulate the activity of the same brain systems. According to the phonological loop model, one would expect that, during memorizing, the differences between ERPs to letters accompanied by different degrees of an irrelevant speech effect and between ERPs to letters differing in the degree of phonological similarity should yield comparable topographical results.

A possible conceptual framework for the ideas outlined above is the so-called state trace analysis proposed by Bamber (1979). This approach postulates the existence of latent variables-that is, hypothetical unobservable variables linking independent and dependent variables. A state trace is a graph that plots the value of one dependent variable as a function of a second dependent variable. State traces may be used to test theories concerning the mechanisms by which independent variables affect dependent variables and whether these mechanisms differ among several independent variables.

If this terminology is applied to Baddeley's model of working memory, the independent variables irrelevant speech and phonological similarity both affect a common latent variable that might be termed "confusion within the phonological short-term store." The dependent variables are memory performance in terms of error rates and - specific to the present study - the topographical ERP pattern. This concept is presented in Figure 1. Figure la shows the general theoretical structure underlying state trace analysis, and Figure $\mathrm{lb}$ represents the application of this general theoretical structure to the specific variables of the present study.

Within this framework, Baddeley's model of working memory predicts that the state traces-that is, the functions relating the dependent variables in the presence of either a low or a high irrelevant speech effect-are identical to those in the presence of either a low or a high phonological similarity effect. In other words, the phonological loop model predicts the same state traces-that is, the same relationship between ERP pattern and memory performance- -when either irrelevant speech or phonological similarity effects are manipulated. If, by contrast, a given dependent variable such as the ERP pattern showed qualitatively different results when one compares irrelevant speech and phonological similarity effects, 


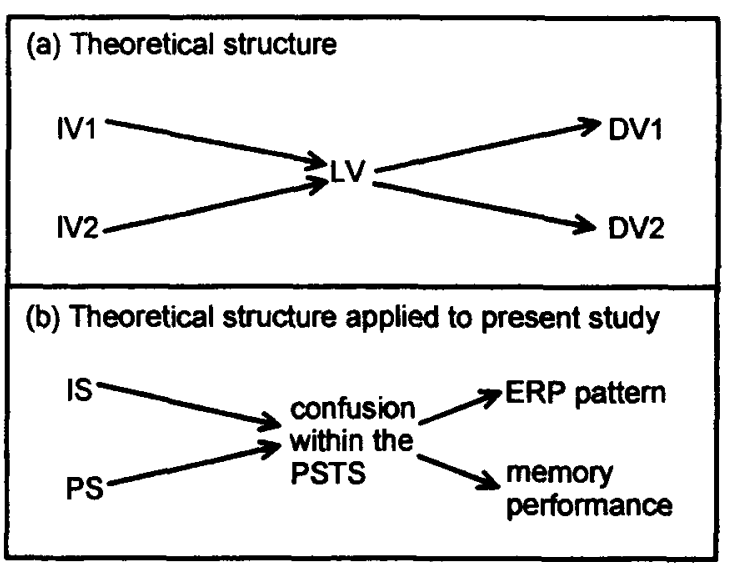

Figure 1. Formalisms of the state trace analysis. (a) is the representation of a general theoretical structure of the type used in the present study. IVI and IV2, independent variables; LV, latent variable; DV1 and DV2, dependent variables. (b) applies the theoretical structure above to the variables used in the present study. IS, irrelevant speech effect; PS, phonological similarity effect; PSTS, phonological short-term store.

the state traces would not be identical and, therefore, would contradict the phonological loop model.

In the present study, we used auditory presentation of consonants. In order to obtain clean ERPs to the memorized stimuli (targets), these stimuli were always alternated with irrelevant stimuli (nontargets). Targets and nontargets were spoken by different voices. Irrelevant speech and phonological similarity effects were imposed on this basic structure by manipulating the phonological similarity between and across targets and nontargets.

In a control condition (Condition 1), target stimuli were phonologically different from one another $(J, Q, S$, $V, X, Z)$, whereas nontarget letters were phonologically dissimilar to the targets, but similar to one another $(B, C$, $D, G, P, T)$. According to both Baddeley's and Jones's models, the irrelevant speech effect should be weak or absent here, and at the same time there should be no or very little phonological similarity effect.

To obtain an irrelevant speech effect in Condition 2, the same phonologically dissimilar letters were used as both targets and nontargets. With this procedure, an irrelevant speech effect should be present relative to Condition 1 according to Baddeley's model due to the phonological similarity between targets and nontargets. Condition 2 would also be regarded as an irrelevant speech condition in Jones's model because the nontarget stimuli were highly variable.

For the phonological similarity effect, target letters that were phonologically similar to one another $(B, C, D$, $G, P, T)$ were used. Interestingly, however, the models of Baddeley and Jones diametrically disagree as to which kind of nontarget letters would yield a pure phonological similarity condition and which would produce a combination with the irrelevant speech effect. Hence, it was decided to use two different phonological similarity conditions. In Condition 3, the nontarget stimuli were the letters
$J, Q, S, V, X, Z$, which are phonologically different from the targets. This should be a rather pure phonological similarity condition according to Baddeley's model, since nontarget letters should not cause an irrelevant speech effect. According to Jones's model, Condition 3 should yield both irrelevant speech and phonological similarity effects because, in addition to the phonological similarity of the targets, the nontargets show phonological variability. In Condition 4 , the nontargets were the letters $B, C, D, G, P, T$, which are phonologically similar to one another. This should be a pure phonological similarity condition according to Jones's model, since nontarget letters should cause no or a very diminished irrelevant speech effect. Conversely, Condition 4 should elicit both effects according to Baddeley, given that in addition to the phonological similarity of the target stimuli, the nontargets are also phonologically similar to the targets. The different conditions are summarized in Table 1.

As a second aim of our study, the opposite predictions yielded by the two models as to which condition would yield the combined effects of irrelevant speech and phonological similarity were tested. If both effects are additive at the performance level (Jones \& Macken, 1995; Salamé \& Baddeley, 1986), the comparisons between Conditions 3 and 4 should indicate which condition combines both effects (i.e., the condition with the higher error rates). Additionally, the ERPs that according to each model should yield the combined effects were compared to the sum of those ERPs that should represent the individual effects. Determining which comparison shows a better fit should also shed some light on the controversy about the mechanism of the irrelevant speech effect.

\section{METHOD}

\section{Subjects}

Twelve subjects ( 7 female), ranging in age between 20 and 41 years $(M=26)$, participated in the study. Subjects reported no neurological or hearing complaints. All subjects were right-handed, with average handedness scores (Oldfield, 1971) of +.82 , with a range of +.60 to +.100 . Subjects were paid a nominal hourly amount.

\section{Stimuli}

Stimuli consisted of two pools of auditorily presented consonant letters that were monosyllabic when pronounced in German. One pool was composed of phonologically similar letters $(B, C, D, G, P$, $T$ ), and the other was composed of phonologically dissimilar letters $(J, Q, S, V, X, Z)$. The phonologically dissimilar letters were dis-

Table 1

Experimental Conditions

\begin{tabular}{|c|c|c|}
\hline Condition & Targets & Nontargets \\
\hline 1 (Control) & Dissimilar & Similar \\
\hline 2 (Irrelevant speech) & Dissimilar & Dissimilar \\
\hline $\begin{array}{l}3 \text { (Phonological similarity plus } \\
\text { irrelevant speech [Jones]) } \\
4 \text { (Phonological similarity nlus }\end{array}$ & Similar & Dissimilar \\
\hline $\begin{array}{l}\text { (Pnonological similarity plus } \\
\text { irrelevant speech [Baddeley]) }\end{array}$ & Similar & Similar \\
\hline
\end{tabular}




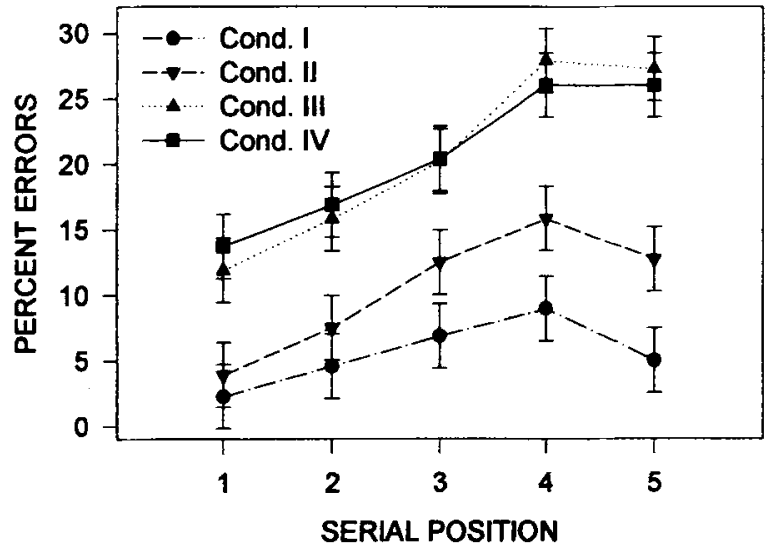

Figure 2. Performance results, expressed as the percentages of errors per condition and serial position. Vertical bars represent confidence intervals for a $95 \%$ criterion. Condition 1 is a control condition. Condition 2 introduces irrelevant speech. Condition 3 represents phonological similarity alone according to Baddeley, but with irrelevant speech according to Jones. Condition 4 represents phonological similarity alone according to Jones, but with irrelevant speech according to Baddeley.

similar not only to one another but also to all the phonologically similar letters.

Both letter pools were recorded when spoken by both a female and a male native German speaker. The male-voice letters were used as targets, and the female-voice letters were used as nontargets. The letters were audiodigitized with a sampling rate of $20 \mathrm{kHz}$, edited to a unitary duration of $280 \mathrm{msec}$ and the same average intensity, and stored on the hard disk of an Amiga $2000 \mathrm{mi}-$ crocomputer system. Each letter could be individually presented by means of headphones. Intensity was adjusted for each subject to a comfortable and clear listening level.

\section{Procedure}

The subjects were seated in an electrically shielded, soundattenuated chamber where a masking noise of $38 \mathrm{~dB}(\mathrm{~A})$ was provided by ventilation. During the presentation of the stimuli, a small cross on a monitor in front of the subjects served as a fixation point. In ERP studies, a fixation point is necessary in order to minimize eye movements, which may cause serious recording artifacts. A chinrest was used to stabilize head position.

Stimuli were presented one by one. Each sequence consisted of 5 target (male voice) and 6 nontarget (female voice) letters. Within each sequence, target and nontarget items alternated, the first and the last items always being a nontarget. The sequence scheme was as follows: N-T-N-T-N-T-N-T-N-T-N, where $\mathrm{N}$ is a nontarget and $\mathrm{T}$ is a target. The interval from nontarget onset to target onset was $1,500 \mathrm{msec}$, and from target onset to nontarget onset, the interval was $1,000 \mathrm{msec}$. This design was preferred to a simultaneous presentation of targets and nontargets because a pilot study showed that when both are presented auditorily they can hardly be identified. Simultaneous dichotic presentation, as used by Hanley and Broadbent (1987), was discarded in order to avoid undesired ERP hemispheric differences unrelated to the effects of interest. The five targets of each sequence were drawn at random without repetition from the six items of either the phonologically dissimilar pool or the phonologically similar pool. The six nontargets consisted of the complete pool of either phonologically dissimilar or phonologically similar items, but were ordered randomly for each sequence. In order to avoid first-order priming effects between nontarget and target items, a nontarget item could not be the same letter as the immediately preceding or following target item.

Four different conditions were obtained by combining the two pools of letters. The combinations have been described in the introduction and represented in Table 1. Forty sequences of each condition (a total of 160 sequences) were presented. The order of conditions was balanced across subjects according to a Latin square. Prior to the experimental series, practice trials were performed consisting of two sequences per each of the four conditions.

Instructions were always given in writing. Subjects were advised to attend only to the male voice, disregarding the female voice, and to memorize the items in their order of presentation. About $770 \mathrm{msec}$ after the onset of the last nontarget, a tone of $60-\mathrm{msec} \mathrm{du}-$ ration and $1000 \mathrm{~Hz}$ advised the subjects to report the sequence of targets. During the response period, subjects were supposed to report each letter in its order of presentation (serial recall). If any letter had been forgotten, they were supposed to say, "I don't know." Subjects were allowed to correct their responses before passing to the next sequence. If the duration of the response period exceeded $10 \mathrm{sec}$, subjects were encouraged to answer faster. In order to avoid speech artifacts in the EEG (Szirtes \& Vaughan, 1977), subjects were asked to reduce movements of mouth and tongue as much as possible during memorizing, but were allowed to use covert rehearsal. Responses were scored on-line, but were also recorded on tape for later checks. An error score was calculated for each condition and each of the five serial positions of the sequences. After the recording session, subjects were debriefed about their cognitive strategies to memorize information - whether they had used an auditory/verbal code or converted the items to a visual code.

Electrophysiological recordings. The EEG from 20 electrodes (Fz, Cz, Pz, F3, F4, F7, F8, Fp1, Fp2, C3, C4, P3, P4, O1, O2, T3, $\mathrm{T} 4, \mathrm{~T} 5, \mathrm{~T} 6$, and $\mathrm{A} 1$, the left upper mastoid) were commonly referenced to the upper right mastoid (A2). The electrooculogram (EOG) was obtained from below the right eye versus FP2 (vertical EOG) and the left versus right lateral orbital rim (horizontal EOG). Electrode impedances were kept below $10 \mathrm{k} \Omega$. In order to measure tongue or mouth movements, an additional electrode was located on the submandibular surface and was differentially amplified against an electrode located on the right massetter muscle. This placement was chosen because it had been shown to be the most sensitive to subvocal articulation in preliminary recordings.

The EEG and EOG were amplified with a bandpass of .03$40 \mathrm{~Hz}$ - high-frequency filter was $1000 \mathrm{~Hz}$ for submandibular/massetter activity - and sampled at a rate of $100 \mathrm{~Hz}$ for $2 \mathrm{sec}$, starting

Table 2

Results of the Analysis of Variance on Event-Related Potential Amplitudes

\begin{tabular}{|c|c|c|c|c|c|c|c|c|c|c|c|c|c|c|c|}
\hline \multirow[b]{3}{*}{ Source } & \multirow[b]{3}{*}{$d f \mathrm{~s}$} & \multicolumn{14}{|c|}{ Time Window (in Milliseconds) } \\
\hline & & \multicolumn{2}{|c|}{$60-100$} & \multicolumn{2}{|c|}{$150-200$} & \multicolumn{2}{|c|}{$200-300$} & \multicolumn{2}{|c|}{$300-400$} & \multicolumn{2}{|c|}{$420-720$} & \multicolumn{2}{|c|}{$730-1,000$} & \multicolumn{2}{|c|}{$1,010-1,510$} \\
\hline & & $F$ & $M S_{\mathrm{c}}$ & $F$ & $M S_{\mathrm{e}}$ & $F$ & $M S_{\mathrm{e}}$ & $F$ & $M S_{\mathrm{e}}$ & $F$ & $M S_{\mathrm{e}}$ & $F$ & $M S_{\mathrm{e}}$ & $F$ & $M S_{\mathrm{e}}$ \\
\hline $\mathrm{C} \times \mathrm{E}$ & 60,660 & 1.0 & 0.5 & $1.7^{*}$ & 0.9 & $5.1 \S$ & 1.1 & $3.8 \S$ & 1.4 & $1.8+$ & 1.5 & 1.2 & 1.6 & 1.6 & $\overline{2.0}$ \\
\hline$P \times E$ & 40,440 & $3.0 \ddagger$ & 0.6 & $4.8 \S$ & 0.8 & $3.4_{+}^{+}$ & 1.3 & 1.2 & 2.1 & 1.5 & 2.4 & 0.9 & 3.9 & 1.3 & 5.2 \\
\hline$C \times P \times E$ & 120,1320 & 0.5 & 0.6 & 0.9 & 0.7 & 1.2 & 0.8 & 1.0 & 1.0 & 0.8 & 1.0 & 1.1 & 1.5 & 1.1 & 1.8 \\
\hline
\end{tabular}

Note-C, condition; E, electrode; P, serial position. $\quad{ }^{*} p<.1 . \quad+p<.05 . \quad+p<.01 . \quad \$ p<.001$. 


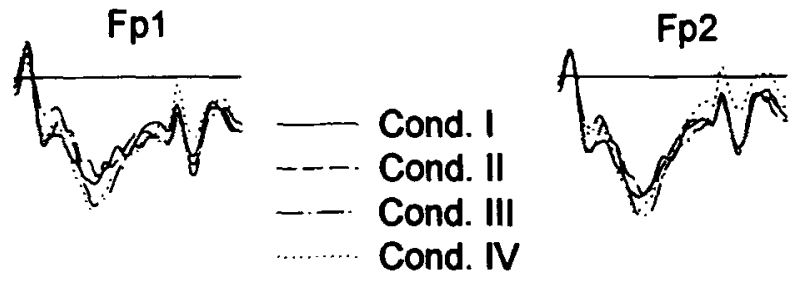

F7

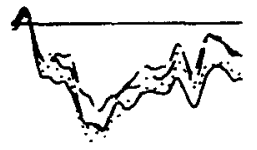

T3
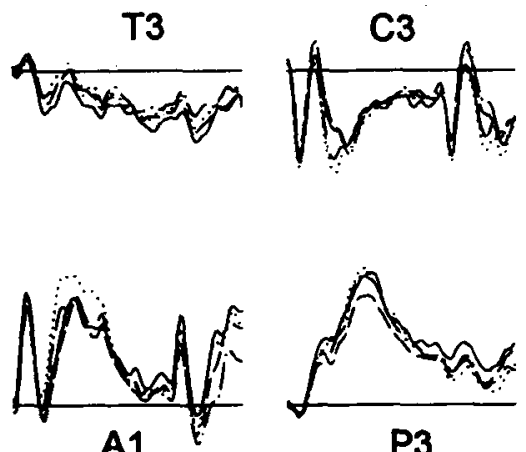

A1

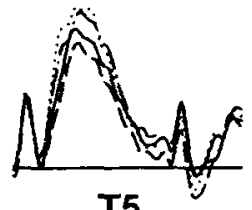

T5
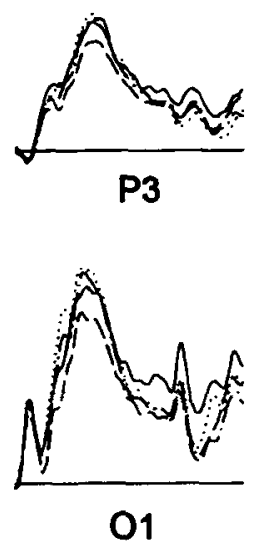

Fz

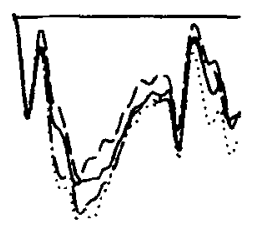

F4
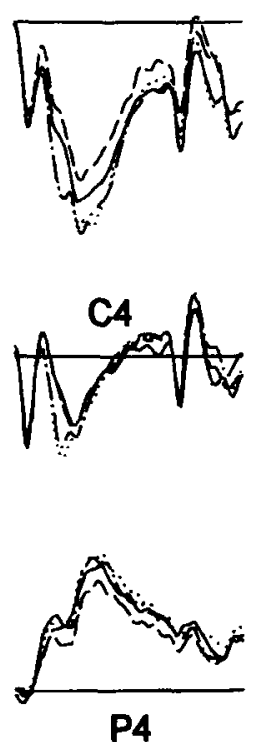

Pz
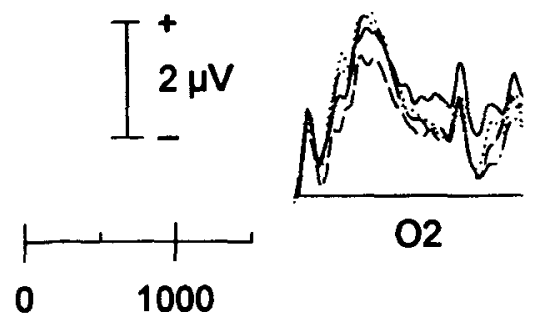

$\mathrm{O} 2$
F8
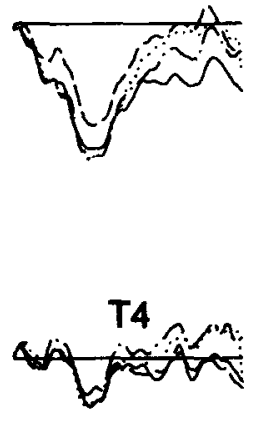

msec

Figure 3. Topographical display of event-related potentials for the four conditions, collapsed over serial positions. Target onset was at the 0 -msec point, and the nontarget occurred at $1,000 \mathrm{msec}$. Condition 3 represents phonological similarity alone according to Baddeley, but with irrelevant speech according to Jones. Condition 4 represents phonological similarity alone according to Jones, but with irrelevant speech according to Baddeley.

430 msec prior to target onset. Thus, each epoch covered a pretarget baseline of $430 \mathrm{msec}$, the whole target-nontarget interval, and $570 \mathrm{msec}$ of the response to the following nontarget. The ERPs to the first nontarget in each sequence were discarded. Ocular artifacts were corrected off-line with the method described by Elbert, Lutzenberger, Rockstroh, and Birbaumer (1985). Trials with uncorrectable EOG artifacts or with submandibular/massetter activity exceeding $100 \mu \mathrm{V}$ were excluded from analysis. ERPs were sorted and averaged separately for each condition and for Serial Positions 1, 2, 4, and 5 . Averaging across Positions $2-4$ was necessary due to capacity limitations of the university mainframe computer for performing analyses of variance (ANOVA). After averaging, the ERPs were recalculated to an average reference derivation (Lehmann, 1987). According to the main components in the ERP waveforms, the epochs were divided into seven time windows. The first one comprised $200 \mathrm{msec}$ before stimulus onset and was used as baseline. The other windows (in milliseconds) were $60-90$ ( $\mathrm{N} 100$ component), 150-200 (P200), 200-300, 300-400, 420-720, 730-1,000, and 1,010-1,510, with the latter covering much of the response to the nontargets.

\section{RESULTS}

\section{Performance}

The performance in the different conditions and for the serial positions is shown in Figure 2. Given that Baddeley's and Jones's models differ as to which effects are present in Conditions 3 and 4 , a $2 \times 2$ design with irrel- 
evant speech and phonological similarity as orthogonal factors was considered inappropriate. Thus, confidence intervals (Loftus \& Masson, 1994) were calculated in order to assess the magnitude of the differences between conditions (Figure 2). They revealed that Conditions 1 and $2(5.5 \%$ vs. $10.5 \%$ errors, respectively) differed significantly, indicating an irrelevant speech effect. Both conditions showed an overlap at Positions 1 and 2 but not at higher positions, which indicates an interaction between condition and serial position for the irrelevant speech effect. Conditions 3 and 4 were both significantly different from Condition 1. Both Conditions 3 and 4 also differed significantly from Condition 2, but did not differ from each other. In fact, the percentage of errors in Conditions 3 and 4 was identical $(20.6 \%)$. This suggests a strong effect of phonological similarity but no effect of irrelevant speech when it is presented together with phonological similarity, regardless of whether one would use Baddeley's or Jones's model to determine which condition combines both effects. There was also a strong serial position effect consisting in a monotonic increase of the error rates from Position 1 to 4 and a decrease for Position 5, reflecting primacy and recency effects, respectively.

The majority of subjects ( 10 out of 12 ) reported purely auditory strategies for Conditions 1 and 2 . One subject reported a mixed strategy for these conditions, and 1 subject reported a purely visual code. The same strategies were also used for Conditions 3 and 4 except for 2 subjects who switched from purely auditory to mixed auditory/visual strategies.

\section{Event-Related Potentials}

Figure 3 shows the ERPs for the four conditions, collapsed over serial positions. As expected, ERPs to targets as well as to nontargets showed $\mathrm{N} 1$ and $\mathrm{P} 2$ components at about 80 and $180 \mathrm{msec}$, respectively, maximal at vertex $(\mathrm{Cz})$. There was also a later component of high amplitude to the relevant stimuli, with positive and negative polarity at posterior and frontal electrodes, respectively.

ERPs for the experimental conditions showed the same general characteristics as those for the control condition. The modulations of the basic waveshape by experimental conditions are depicted in Figure 4, which shows the difference waves - that is, the results of subtracting the ERPs of the control condition (1) from the different experimental conditions.

The difference waves of Condition 2-1, representing the irrelevant speech effect, showed a long-lasting activity with positive values over frontal electrodes and negative values over posterior and occipital electrodes. This activity was bilaterally distributed. The difference waves Conditions 3-1 and 4-1, representing the phonological similarity effect-with or without irrelevant speech effect-were quite similar. They showed pronounced peaks with negative values around $290 \mathrm{msec}$ at fronto-central electrodes and with a reversal of polarity at parietotemporal and occipital electrodes. This component presents larger amplitudes over the right hemisphere for fronto-central electrodes and over the left for parietotemporal and occipital electrodes. Because the ERP pattern in Condition 3-1 is quite similar to that in Condition 4-1, it appears to be mainly the result of phonological similarity. The main difference between Conditions 3-1 and 4-1 seems to be larger amplitudes of this pattern, mainly over left-hemisphere electrodes for Condition 4-1.

Separate repeated measures ANOVAs were applied to the average amplitudes of the ERPs of each time window. As for the performance data, a $2 \times 2$ design with irrelevant speech and phonological similarity as orthogonal factors was considered inappropriate given that Baddeley's and Jones's models differ as to which effects are present in Conditions 3 and 4 . Thus, a two-way repeated measures ANOVA was used to assess the effects of condition (four levels), serial position (three levels), and electrode (21 levels). Factor serial position covered the three levels used for ERP averaging. Significance levels of the ANOVA results were calculated after correction of the degrees of freedom for violations of the sphericity assumption with the Huynh-Feldt method. Post hoc comparisons were performed by means of pairwise ANOVAs corrected with the Bonferroni method.

Table 2 shows the results of the ANOVA and Table 3 shows the post hoc comparisons for the ERPs. Significant condition effects were present for the time windows between 200 and $700 \mathrm{msec}$. Post hoc comparisons for the 200- to 300-msec window showed that Conditions 3 and 4 did not differ significantly, whereas all other comparisons were significant. In the 300 - to 400 -msec window, Condition 4 was significantly different from Conditions 2 and 3 . In the 420 - to 720 -msec window, only Conditions 2 and 4 differed significantly.

These results indicate that the long-lasting activity of irrelevant speech alone (Condition 2-1) was most reliable in the 200- to $300-\mathrm{msec}$ window. The phonological similarity conditions ( 3 and 4 ) did not differ significantly from each other but both were significantly different from the other conditions at the 200- to $300-\mathrm{msec}$ window, covering the 290 -msec peak found for this effect. The ERP differences between Conditions 3 and 4 in the 300 - to 400 -msec time windows clearly indicate that the ERP results are not fully in parallel with the performance results. Whereas the performance data showed no irrelevant speech effect when phonological similarity

Table 3

Post Hoc Comparisons of Event-Related Potential Amplitudes Between Experimental Conditions

\begin{tabular}{|c|c|c|c|c|c|c|}
\hline \multirow{3}{*}{$\begin{array}{l}\text { Condition } \\
\text { Comparison }\end{array}$} & \multicolumn{6}{|c|}{ Time Window (in Milliseconds) } \\
\hline & \multicolumn{2}{|c|}{$200-300$} & \multicolumn{2}{|c|}{$300-400$} & \multicolumn{2}{|c|}{$420-720$} \\
\hline & $F$ & $M S_{\mathrm{e}}$ & $F$ & $M S_{\mathrm{e}}$ & $F$ & $M S$ \\
\hline 1 vs. 2 & $3.9^{*}$ & 0.9 & 2.5 & 1.7 & 1.5 & 1.7 \\
\hline l vs. 3 & $5.4^{*}$ & 0.9 & 1.2 & 1.5 & 0.7 & 1.2 \\
\hline I vs. 4 & 4. $1^{*}$ & 1.2 & 2.7 & 1.5 & 1.0 & 1.8 \\
\hline 2 vs. 3 & $8.1 \dagger$ & 1.0 & 4.3 & 1.1 & 2.6 & 1.3 \\
\hline 2 vs. 4 & $7.1 \dagger$ & 1.6 & $8.9+$ & 1.5 & $4.4^{*}$ & 1.6 \\
\hline 3 vs. 4 & 1.4 & 1.2 & $3.2^{*}$ & 0.9 & 0.8 & 1.4 \\
\hline
\end{tabular}




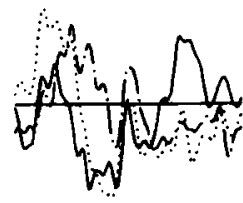

Fp1

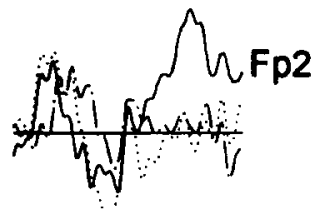

.

Cond. III-I

Cond. IV-I
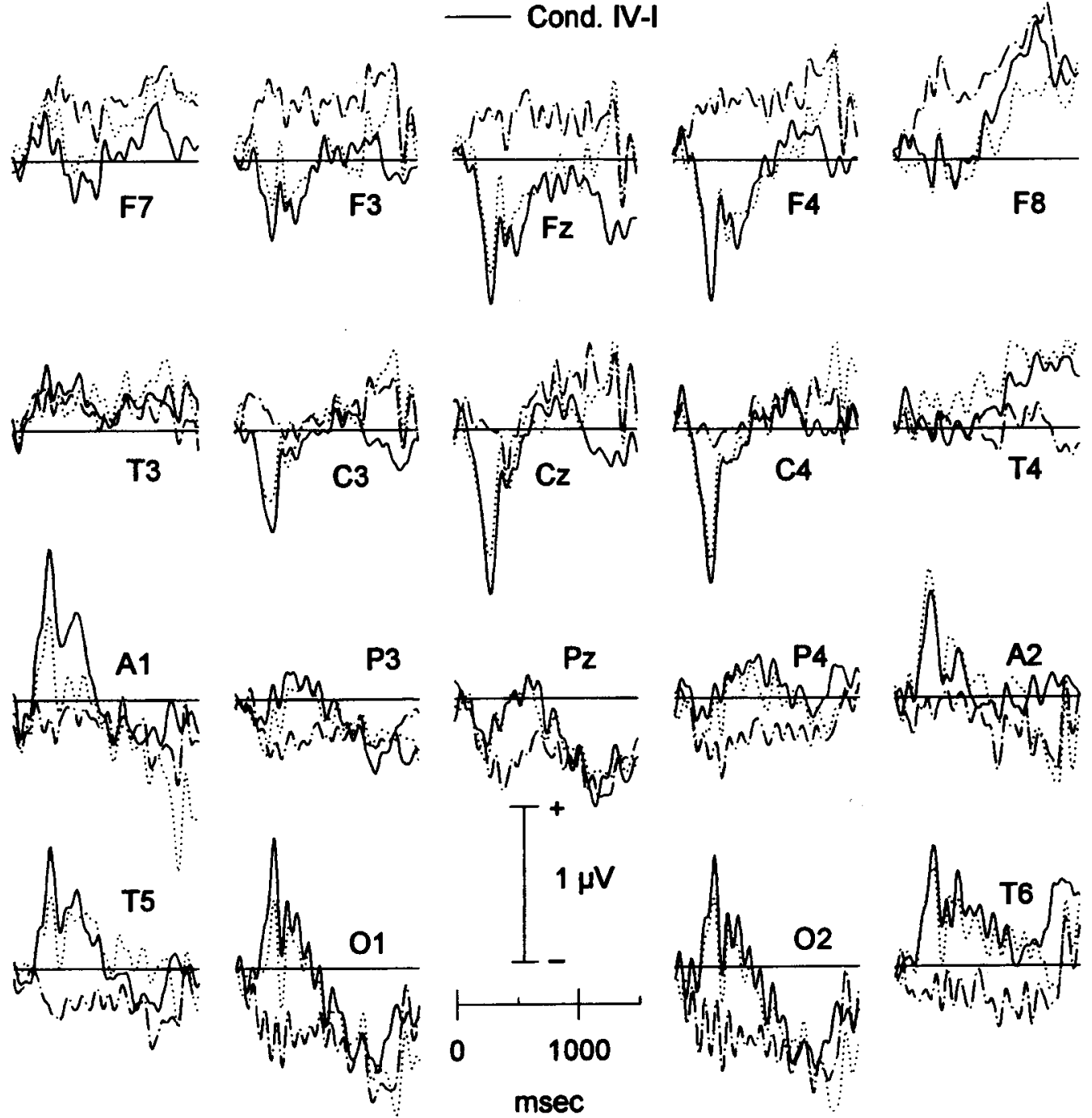

Figure 4. Difference waves representing the effects of irrelevant speech (Condition 2-1) and phonological similarity (Conditions 3-1 and 4-1). Note the difference in the $\mu \mathrm{V}$ scale between this figure and the previous one.

was present, the ERP data showed that irrelevant speech causes some additional activity not present in the pure phonological similarity condition. Thus, even without defining which condition combines both effects, it can be concluded that irrelevant speech does influence brain activity under phonological similarity conditions.

When experimental effects on ERP amplitudes depend on electrode position, as was the case here, it may relate to either differences in the ERP-generating brain structure or to different degrees of activity of the same set of brain structures (McCarthy \& Wood, 1985). In order to elucidate whether the observed ERP differences between the experimental conditions were due to genuine differences in generating structures, amplitude differences were eliminated by scaling (profile analysis). In order to isolate effects of the experimental manipulations, the profile analyses were based on the difference waves. For each time window of the difference waves, mean amplitudes were scaled for each subject across all electrodes, with the average distance from the mean, calculated from the grand mean ERPs, as divisor. Significant differences in ANOVAs with these scaled data, where 

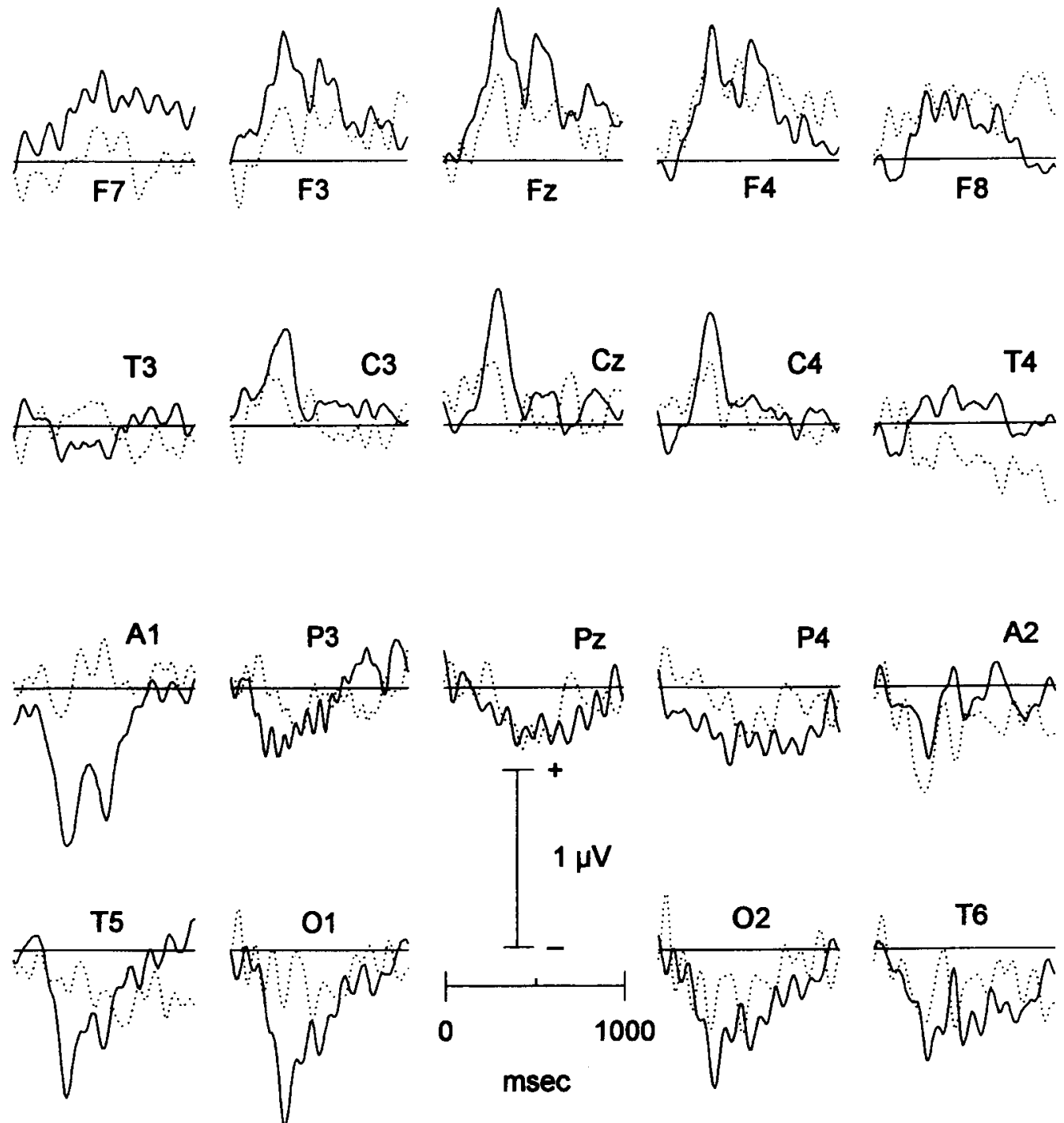

Figure 5. Residual event-related potential (ERP) waveshapes resulting from the subtraction of ERPs that should be equivalent according to either Baddeley's or Jones's model of irrelevant speech.

possible effects of source strength are eliminated, provide unambiguous evidence for different brain sources.

In the 200- to $300-\mathrm{msec}$ window, the Difference Waves 2-1 showed a different topography from both 3-1 $\left[F(20,220)=7.8, M S_{\mathrm{e}}=0.1, p<.01\right]$ and $4-1[F(20,220)$ $\left.=7.1, M S_{\mathrm{e}}=0.2, p<.01\right]$, whereas the latter did not differ from each other. This indicates that phonological similarity, whether alone or together with irrelevant speech, causes a topographical pattern significantly different from that of irrelevant speech. For the remaining time windows, there were topographical differences only between Conditions 2- 1 and $4-1\left[F(20,220)=8.9, M S_{\mathrm{e}}=\right.$ $0.1, p<.01]$ for the $300-$ to $400-\mathrm{msec}$ window, and $\left[F(20,220)=4.1, M S_{\mathrm{e}}=0.2, p<.05\right]$ for the $420-$ to 720-msec window.

Whereas performance data did not distinguish between the condition combining both irrelevant speech and phonological similarity effects and the one presenting only the phonological similarity effect, the ERP differences observed between Conditions 3 and 4 may be 
used for this purpose. It appears plausible to interpret the ERP differences between Conditions 3 and 4 by assuming that an irrelevant speech effect is added to the phonological similarity effect in one of these conditions. However, as explained in the introduction, the predictions made by Baddeley's and Jones's models are different. Baddeley's model predicts

$$
(4-1) \approx(2-1)+(3-1),
$$

whereas Jones's model predicts

$$
(3-1) \approx(2-1)+(4-1) \text {, }
$$

where 1 to 4 are the ERPs from the different conditions. Figure 5 illustrates the fit of these predictions with the data - that is, the difference waves $[(4-1)]-[(2-1)+(3-$ 1)] (Baddeley) and [(3-1)] - [(2-1) + (4-1)] (Jones). The smaller the residual, the better the fit of the data with the model. Overall, it appears that Jones's model is a better approximation to the data than is Baddeley's model. This impression was statistically verified by comparing the composite amplitudes at the two sides of Equations 1 and 2 by means of repeated measures ANOVAs for the different time windows (Table 4). Significant effects indicate that the ERPs contradict the model.

Clearly, the ERPs are at variance with Baddeley's model. In contrast, there is no obvious conflict between the observed and the predicted ERPs according to Jones's model. However, pure summation of Conditions 1 and 4, as predicted by Jones, does not explain all activity in Condition 3, as indicated by two statistical trends in Table 4 . As noted, Conditions 3 and 4 did not differ over the right hemisphere, whereas the effects of irrelevant speech alone (Condition 2) were bilaterally distributed. Figure 5 shows that left-hemisphere data approach the zero line better than those on the right side. Hence, summation of both effects appears to be true mainly for the left hemisphere, whereas irrelevant speech seems to cause only a very weak, if any, effect over the right hemisphere when phonological similarity is present.

Table 2 shows that serial position effects were confined to the early portions of the ERPs $(60-300 \mathrm{msec})$ and did not interact with condition. These effects consisted in a monotonic decrease in ERP amplitude over positions for that time range. This might reflect sensory habituation or refractoriness, as would be expected especially for the N1 component (Näätänen, 1992). An

Table 4

Testing the Fit of Irrelevant Speech Models With Event-Related Potential Amplitudes

\begin{tabular}{cccccc}
\hline \multirow{2}{*}{$\begin{array}{c}\text { Time Window } \\
\text { (in Milliseconds) }\end{array}$} & \multicolumn{2}{c}{ Baddeley's Model } & & \multicolumn{2}{c}{ Jones’s Model } \\
\cline { 2 - 3 } \cline { 5 - 6 } & $F$ & $M S_{\mathrm{e}}$ & & $F$ & $M S_{\mathrm{e}}$ \\
\hline $150-200$ & 1.2 & 0.2 & 1.6 & 0.2 \\
$200-300$ & $3.4 \ddagger$ & 0.2 & $1.9^{*}$ & 0.2 \\
$300-400$ & $2.2^{*}$ & 0.3 & 1.2 & 0.2 \\
$420-720$ & $2.9 \dagger$ & 0.1 & $2.4^{*}$ & 0.3 \\
$730-1,000$ & 1.1 & 0.1 & 0.9 & 0.1 \\
$1,010-1,510$ & 1.4 & 0.3 & 1.5 & 0.2 \\
\hline${ }^{*} p<.1 . \quad \dagger p<.05$. & $\ddagger p<.001$. & $d f \mathrm{~s}=20,220$. & &
\end{tabular}

early sensory interpretation of the serial position effects is also compatible with the fact that their onset occurred at a time when stimulus analysis could not have been completed.

Finally, a two-way repeated measures ANOVA was applied to the submandibular/massetter recordings with the same time windows, factors (except for electrode sites), and procedures as those used for the ERPs. Post hoc comparisons showed differences only for the 150 - to 200-msec window between Conditions 3 and $4[F(1,11)=$ $\left.12.3, M S_{\mathrm{e}}=3.6, p<.05\right]$ and for the $300-$ to $400-\mathrm{msec}$ window between Conditions 1 and $4[F(1,11)=11.3$, $\left.M S_{\mathrm{e}}=4.2, p<.05\right]$. These significant differences do not coincide with the observed ERP effects. Thus, an explanation of the relevant ERP results by influences of mouth movements is implausible.

\section{DISCUSSION}

\section{Performance}

As expected, both irrelevant speech and phonological similarity affected recall, but to different degrees. Irrelevant speech significantly increased error rates, although weakly, whereas phonological similarity had a much more marked influence. However, considering the similarity of Conditions 3 and 4 in terms of error rates, phonological similarity and irrelevant speech effects do not seem to be additive, as has been reported for sequences of less than eight items (Jones \& Macken, 1995; Salamé \& Baddeley, 1986). Instead, regardless of which of our conditions represents the combined effects, our results appear to indicate no significant irrelevant speech effect when memorized items are phonologically similar.

Interactions of irrelevant speech and phonological similarity have been reported for eight-item sequences. But contrary to our results, for these kinds of sequences there was no effect of phonological similarity (Colle \& Welsh, 1976; Salamé \& Baddeley, 1986). Interestingly, however, in reports about five-item sequences, the figures seem to indicate interactions of irrelevant speech and phonological similarity, whereas the corresponding ANOVAs always indicate additive effects. Therefore, the contradiction between our results and those of previous studies might merely be a consequence of our data analysis procedure. Therefore, a $2 \times 2$ ANOVA was performed on the error rates. The ANOVA also appeared to be more justified because the ERP results (see below) indicated that Condition 3 may combine both irrelevant speech and phonological similarity effects, whereas Condition 4 represents phonological similarity alone.

The ANOVA indicated significant main effects of irrelevant speech $\left[F(1,11)=5.6, M S_{\mathrm{e}}=10.4, p<.05\right]$ and phonological similarity $\left[F(1,11)=26.4, M S_{\mathrm{e}}=57.5\right.$, $p<.001]$, but no interaction $\left[F(1,11)=1.8, M S_{\mathrm{e}}=31.9\right.$, $p>.1]$. Interestingly, our performance results are now in line not only with those of Salamé and Baddeley (1986) and Jones and Macken (1995), but, strikingly, also with our ERP results, indicating an additivity of irrelevant speech and phonological similarity. 
The serial position effects found here are in line with previous findings (Colle, 1980; Colle \& Welsh, 1976; Salamé \& Baddeley, 1982), with error rates increasing over positions except for a slight recency effect. Consistent with occasional reports of an interaction: of serial position and irrelevant speech (Jones \& Macken, 1995), confidence intervals (see Figure 2) for Conditions 1 and 2 suggest somewhat larger differences at later positions. It may also be noted that effects of strategies for memorizing the items on these findings should be marginal, since the majority of our subjects invariably used an auditory code.

The idea that our design might have induced a "sandwhich effect" (Baddeley, Papagno, \& Andrade, 1993) instead of irrelevant speech, by interpolating irrelevant speech between relevant material, can be discarded. We found significant differences between Conditions 1 and 2 , which differ in the degree of variability of irrelevant material and in the degree of phonological similarity between targets and nontargets-precisely the two variables that, according to either Baddeley's or Jones's model, explain the irrelevant speech effect, but to which the sandwhich effect is insensitive (Baddeley et al., 1993). The main difference between our design and the sandwich procedure is that we did not use the standard rhythmic alternation of targets and nontargets characteristic of sandwich paradigms. Salamé and Baddeley (1982), Miles et al. (1991), and LeCompte (1994) showed that the simultaneous presentation of relevant and irrelevant material is not mandatory to obtain an irrelevant speech effect.

\section{Event-Related Potentials}

The main aim of our study was to elucidate whether the two effects that, according to the current phonological loop model, influence the phonological short-term store, elicit ERP effects with similar topographies. The profile analysis confirmed that in the time window in which both effects were most reliable $(200-300 \mathrm{msec})$, there was a significant difference in topography between the two conditions with a phonological similarity effect and the condition with an irrelevant speech effect, whereas both conditions with a phonological similarity effect failed to differ. It is also remarkable that the most reliable ERP effects of both irrelevant speech and phonological similarity were observed in the same (200- to $300-\mathrm{msec})$ time window, which excludes the possibility that both factors cause the same ERP effect but at different time windows. We may therefore conclude that irrelevant speech and phonological similarity effects are modulating the activity of different brain systems.

Given that it seems implausible that one and the same cognitive element is based on the activity of different neural substrates, our findings indicate that both irrelevant speech and phonological similarity effects concern different cognitive elements. This conclusion is at variance with the phonological loop model (Baddeley, 1986, 1990), but may explain why irrelevant speech and phonological similarity effects failed to interact in several studies (Jones \& Macken, 1995; Salamé \& Baddeley, 1986). According to our data, and as developed below in detail, there is no evidence against the phonological short-term store as the locus of action of the irrelevant speech effect. The phonological similarity effect, however, may act, at least partially, on phonological analysis processes.

Unfortunately, it is impossible to plot true state traces between the dependent variable error rates and ERP topography because the latter involves amplitude measures at 21 electrodes. However, the topographies of the difference waves (Figure 4) may be considered as approximations to state traces, given that there are only two levels of performance and that the differences in performance are equal to or least comparable for both irrelevant speech and phonological similarity effects. In this vein, the significantly dissimilar difference waves for the irrelevant speech effect and the phonological similarity effect indicate that the state trace relating ERP topography and memory performance when irrelevant speech is manipulated is different from the state trace under phonological similarity. In other words, at variance with the theoretical structure derived from Baddeley's phonological loop hypothesis, there are different state traces for independent variables irrelevant speech and phonological similarity. This means that the independent variables affect at least one different latent variable. Note, however, that they may also share one or more additional latent variables. As will be detailed below, our data seem to indicate that phonological similarity affects a latent variable that might be termed "confusion during phonological analysis" instead of or in addition to the latent variable affected by irrelevant speech ("confusion within the phonological short-term store").

It appears difficult to relate the irrelevant speech ERP pattern to previous reports, even to those that investigated ERPs in verbal working memory (Barrett \& Rugg, 1990; Lang et al., 1992; Patterson, Pratt, \& Starr, 1991; Pratt, Michalewski, Patterson, \& Starr, 1989; Ruchkin et al., 1992; Ruchkin et al., 1994; Ruchkin, Johnson, Canoune, \& Ritter, 1990; Rugg, 1984a, 1984b). A possible explanation for this is that irrelevant speech has not been previously manipulated in ERP studies. Therefore, it is difficult to directly infer from our data which is the locus of action of the irrelevant speech effect. Hence, there is no evidence to discard the phonological short-term store.

By contrast, there are two major candidates for known ERP components that our phonological similarity effect might be identified with: the "phonological N400" and the "Nd." The phonological N400 has been reported with latencies and topographies similar to our phonological similarity effect, and is considered to reflect the time course of the processing of phonological information (Praamstra, Meyer, \& Levelt, 1994; Praamstra \& Stegeman, 1993). The topographical distribution of our phonological similarity effect is also quite similar to that of the $\mathrm{Nd}$, which is usually observed in selective attention paradigms, where particular stimuli have to be detected in one of two or more different channels (Giard. 
Perrin, Pernier, \& Peronnet, 1988; Näätänen, 1990 1992; Woods, 1990; Woods, Alho, \& Algazi, 1991). In tasks that required a phonological analysis, the $\mathrm{Nd}$ showed latencies similar to those of our phonological similarity effect (Hansen, Dickstein, Berka, \& Hillyard, 1983; Hansen \& Hillyard, 1980; Woods, Hillyard, \& Hansen, 1984). ${ }^{1}$

Hence, regardless of whether our ERP effect of phonological similarity corresponds to phonological N400 or $\mathrm{Nd}$, it appears to reflect the fact that phonologically similar material requires more processing than does dissimilar material. These increased processing demands may concern the extraction of differentiating stimulus features of the memorized items, which are few and difficult to find in phonological similarity conditions. This is in agreement with several reports that stimuli with less discriminable attributes elicit larger Nds (Alho, Sams, Paavilainen, \& Näätänen, 1986; Hansen et al., 1983; Schwent, Hillyard, \& Galambos, 1976; Woods et al., 1984). Thus, according to our results, the phonological similarity effect in memory might be, at least partially, secondary to problems of phonological analysis. Rather than being due to a problem within the phonological short-term store itself, the phonological similarity effect may relate to a degradation of the phonetic trace or a lack of differentiating cues at the level of phonological analysis. In state trace terminology, this result raises the possibility that phonological similarity affects a latent variable related to phonological analysis.

Considering the partially tentative interpretation of the present ERP effects, one might argue that these effects are related not so much to memory, but rather to some other variable. For instance, the higher level of difficulty in the phonological similarity as compared with the irrelevant speech condition could have increased subjects' arousal, frustration, or anxiety; or, alternatively, it might have given rise to qualitatively different processing strategies. Quantitative effects, as in the former case, are a very unlikely source of the highly different ERP topographies observed here. Hence, the differences found between phonological similarity and irrelevant speech must be qualitative in nature. The gross difference in ERPs strongly indicates that both effects involve different brain processes and therefore cognitive elements. This would seem to be relevant not only to the interpretation of the present ERP results, but also whenever phonological similarity or irrelevant speech affects memory performance. For example, if the ERP effects of phonological similarity reflect the activity of a process that is only indirectly affecting memory performance, such an effect should still be taken into account by models of working memory.

In spite of our findings of clearly different ERP effects of irrelevant speech and phonological similarity, it cannot be excluded that the two effects share common loci of action (e.g., the phonological short-term store) that are not reflected in our recordings. For example, such effects may have occurred during retrieval or, more gener- ally, outside our recording epoch. However, because we did find significant ERP effects during our observation interval, at least some of the effects of both irrelevant speech and phonological similarity on brain activity are reflected in our data. Furthermore, our data show that during the recording epoch used here, irrelevant speech and phonological similarity were influencing different brain generators, and hence presumably different cognitive elements. Therefore, regardless of which concrete elements are actually affected, the fact that these elements are different is at variance with models that assume only one and the same element as the locus of action for both effects.

The second aim of our study was to test whether Baddeley's or Jones's model of irrelevant speech would better explain our data. The ERPs that according to Baddeley's model should be equivalent, clearly differed from each other. In contrast, equivalent ERPs according to Jones's model were much more similar. Therefore, it is Condition 3 that appears to combine both irrelevant speech and phonological similarity effects. In this condition, a pattern similar to the irrelevant speech effect of Condition 2 is added to the phonological similarity effect. The resemblance of our results to predictions made by Jones's model suggests that irrelevant speech is based on acoustic differences in the irrelevant stream rather than on the phonological similarity between target and nontarget items.

In this line, our findings clearly indicate that irrelevant speech also influences brain activity under phonological similarity conditions, despite the total absence of a corresponding performance effect. This holds true regardless of which model of irrelevant speech effect is assumed. These findings are compatible with the notion that the irrelevant speech effect relates to an automatic process, possibly the suggested obligatory access of nontarget material to the phonological short-term store (Baddeley, 1986, 1990, 1992b). This process appears to be present in the condition combining both effects and is reflected in ERPs, but does not cause an additional number of errors in memory performance. This might be accounted for by considering that when relevant material has few differential cues, the irrelevant speech is redundant, thus showing low or no disruptive effects, as predicted by the object-oriented episodic record model of Jones and his colleagues (Jones \& Macken, 1995; Jones \& Morris, 1992)

In conclusion, our data indicate that irrelevant speech and phonological similarity effects produce ERP patterns with significantly different topographies. Thus, the effects influence the activity of different brain structures and, hence, they may concern different cognitive elements. Therefore, the results are at variance with the conception that both variables affect only one and the same system - the phonological short-term store. Rather, they suggest that the phonological similarity effect is acting at the level of phonological analysis. In addition, our data suggest that the irrelevant speech effect is based on pho- 
nological variability within the irrelevant stream more than on phonological similarity between relevant and irrelevant material, in line with a recently developed model regarding the mechanism of this effect.

\section{REFERENCES}

Alho, K., Sams, M., Paavilainen, P., \& Näätänen, R. (1986). Small pitch separation and the selective-attention effect on the ERP. Psychophysiology, 23, 189-197.

BADDELEY, A. D. (1966). Short-term memory for word sequences as a function of acoustic, semantic and formal similarity. Quarterly Journal of Experimental Psychology, 18, 362-365.

Baddeley, A. [D.] (1986). Working memory. Oxford: Oxford University Press, Clarendon Press.

BADDELEY, A. [D.] (1990). Human memory. Theory and practice. Hove, U.K.: Erlbaum.

BADDELEY, A. [D.] (1992a). Is working memory working? The Fifteenth Bartlett Lecture. Quarterly Journal of Experimental Psychology 44A, 1-31.

BADDELEY, A. [D.] (1992b). Working memory. Science, 255, 556-559.

BADDELEY, A. [D.] (1992c). Working memory: The interface between memory and cognition. Journal of Cognitive Neuroscience, 4, 281288.

Baddeley, A. [D.] , Papagno, C., \& Andrade, J. (1993). The sandwich effect: The role of attentional factors in serial recall. Journal of Experimental Psychology: Learning, Memory, \& Cognition, 19, 862-870.

Baddeley, A. [D.] , \& SalamÉ, P. (1986). The unattended speech effect: Perception or memory? Journal of Experimental Psychology: Learning, Memory, \& Cognition, 12, 525-529.

BAmBER, D. (1979). State trace analysis: A method of testing simple theories causation. Journal of Mathematical Psychology, 19, 137-181.

BARRETT, S. E., \& RUGG, M. D. (1990). Event-related potentials and the phonological matching of picture names. Brain \& Language, 38 , 424-437.

Belleville, S., Peretz, I., \& Arguin, M. (1992). Contribution of articulatory rehearsal to short-term memory: Evidence from a case of selective disruption. Brain \& Language, 43, 713-746.

COLLE, H. A. (1980). Auditory encoding in visual short-term recall: Effects of noise intensity and spatial location. Journal of Verbal Learning \& Verbal Behavior, 19, 722-735.

COLle, H. A., \& WELSH, A. (1976). Acoustic masking in primary memory. Journal of Verbal Learning \& Verbal Behavior, 15, 17-31.

Elbert, T., Lutzenberger, W., Rockstroh, B., \& Birbaumer, N. (1985). Removal of ocular artifacts from the EEG-A biophysical approach to the EOG. Electroencephalography \& Clinical Neurophysiology, 60, 455-463.

Gathercole, S. E., \& BAdDeley, A. D. (1993). Working memory and language. Hove, U.K.: Erlbaum.

Giard, M. H., Perrin, F., Pernier, J., \& Peronnet, F. (1988). Several attention-related wave forms in auditory areas: A topographic study. Electroencephalography \& Clinical Neurophysiology, 69, 371-384.

HANLEy, J. R., \& BROADBENT, C. (1987). The effect of unattended speech on serial recall following auditory presentation. British Journal of Psychology, 78, 287-297.

Hansen, J. C., Dickstein, P. W., Berka, C., \& Hillyard, S. A. (1983). Event-related potentials during selective attention to speech sounds. Biological Psychology, 16, 211-224.

Hansen, J. C., \& Hillyard, S. A. (1980). Endogenous brain potentials associated with selective auditory attention. Electroencephalography \& Clinical Neurophysiology, 49, 277-290.

JoHNSON, R. (1993). On the neural generators of the P300 component of the event-related potential. Psychophysiology, 30, 90-97.

JONES, D. M. (1994). Disruption of memory for lip-read lists by irrelevant speech: Further support for the changing state hypothesis. Quarterly Journal of Experimental Psychology, 47A, 143-160.

JONES, D. M., \& MACKEN, W. J. (1993). Irrelevant tones produce an irrelevant speech effect: Implications for phonological coding in working memory. Journal of Experimental Psychology: Learning. Memory, \& Cognition, 19, 369-381.
Jones, D. M., \& Macken, W. J. (1995). Phonological similarity in the irrelevant speech effect: Within- or between-stream similarity? Journal of Experimental Psychology: Learning, Memory, \& Cognition, 21, 103-115.

Jones, D., Madden, C., \& Miles, C. (1992). Privileged access by irrelevant speech to short-term memory: The role of changing state. Quarterly Journal of Experimental Psychology, 44A, 645-669.

JONES, D., \& MORRIS, N. (1992). Irrelevant speech and serial recall: Implications for theories of attention and working memory. Scandinavian Journal of Psychology, 33, 212-229.

Lang, W., Starr, A., Lang, V., Lindinger, G., \& Deecke, L. (1992). Cortical DC potential shifts accompanying auditory and visual shortterm memory. Electroencephalography \& Clinical Neurophysiology, 82, 285-295.

LECOMPTE, D. C. (1994). Extending the irrelevant speech effect beyond serial recall. Journal of Experimental Psychology: Learning, Memory, \& Cognition, 20, 1396-1408.

LehmanN, D. (1987). Principles of spatial analysis. In A. S. Gevins \& A. Rémond (Eds.), Handbook of electroencephalography and clinical neurophysiology: Vol. 1. Methods of analysis of brain electrical and magnetic signals (pp. 309-354). Amsterdam: Elsevier.

LofTus, G. R., \& Masson, M. E. J. (1994). Using confidence intervals in within-subject designs. Psychonomic Bulletin \& Review, 1, 476-490.

Longoni, A. M., Richardson, J. T. E., \& Aiello, A. (1993). Articulatory rehearsal and phonological storage in working memory. Memory \& Cognition, 21, 11-22.

Martín-Loeches, M., Schweinberger, S. R., \& Sommer, W. (1997). The phonological similarity effect in working memory: An ERP study comparing auditory and visual input modalities. Manuscript sumbitted for publication.

McCarThy, G., \& Wood, C. C. (1985). Scalp distributions of eventrelated potentials: An ambiguity associated with analysis of variance models. Electroencephalography \& Clinical Neurophysiology, 62, 203-208.

Miles, C., Jones, D. M., \& Madden, C. A. (1991). Locus of the irrelevant speech effect in short-term memory. Journal of Experimental Psychology: Learning, Memory, \& Cognition, 17, 578-584.

NÄÄTÄNEN, R. (1990). The role of attention in auditory information processing as revealed by event-related potentials and other brain measures of cognitive function. Behavioral \& Brain Sciences, 13, 201-288.

NÄÄTÄNEN, R. (1992). Attention and brain function. Hillsdale, NJ: Erlbaum.

OldFIELD, R. C. (1971). The assessment and analysis of handedness The Edinburgh inventory. Neuropsychologia, 9, 97-113.

Papagno, C., \& Vallar, G. (1992). Phonological short-term memory and the learning of novel words: The effect of phonological similarity and item length. Quarterly Journal of Experimental Psychology, 44A, 41-67.

Patterson, J. V., Pratt, H., \& Starr, A. (1991). Event-related potentials correlates of the serial position effect in short-term memory. Electroencephalography \& Clinical Neurophysiology, 78, 424-437.

Praamstra, P., Meyer, A. S., \& Levelt, W. J. M. (1994). Neurophysiological manifestations of phonological processing: Latency variation of a negative ERP component timelocked to phonological mismatch. Journal of Cognitive Neuroscience, 6, 204-219.

Praamstra, P. \& Stegeman, D. F. (1993). Phonological effects on the auditory N400 event-related brain potential. Cognitive Brain Research, 1, 73-86

Pratt, H., Michalewski, H. J., Patterson, J. V., \& Starr, A. (1989). Brain potentials in a memory-scanning task. III. Potentials to the items being memorized. Electroencephalography \& Clinical Neurophysiology, 73, 41-51.

Ruchkin, D., Grafman, J., Krauss, G. L., Johnson, R., Jr., CaNOUNE, H., \& RITTER, W. (1994). Event-related brain potential evidence for a verbal working memory deficit in multiple sclerosis Brain, 117, 289-305

Ruchim, D., Johnson, R., JR., Canoune, H., \& Ritter, W. (1990). Short-term memory storage and retention: An event-related brain potential study. Electroencephalography \& Clinical Neurophysiology, 76, $419-439$ 
Ruchim, D., Johnson, R., Jr., Grafman, J., Canoune, H., \& RitTER, W. (1992). Distinctions and similarities among working memory processes: An event-related potential study. Cognitive Brain Research, 1, 53-66.

RUGG, M. D. (1984a). Event-related potentials and the phonological processing of words and non-words. Neuropsychologia, 22, 435-443.

RUGG, M. D. (1984b). Event-related potentials in phonological matching tasks. Brain \& Language, 23, 225-240.

Salamé, P., \& Baddeley, A. [D.] (1982). Disruption of short-term memory by unattended speech: Implications for the structure of working memory. Journal of Verbal Learning \& Verbal Behavior, 21, 150164.

Salamé, P., \& Baddeley, A. [D.] (1986). Phonological factors in STM: Similarity and the unattended speech effect. Bulletin of the Psychonomic Society, 24, 263-265.

SALAMÉ, P., \& BADDELEY, A. [D.] (1989). Effects of background music on phonological short-term memory. Quarterly Journal of Experimental Psychology, 41A, 107-122.

Schwent, V. L., Hillyard, S. A., \& Galambos, R. (1976). Selective attention and the auditory vertex potential. II. Effects of signal intensity and masking noise. Electroencephalography \& Clinical Neurophysiology, 40, 615-622.

Starr, A., Barrett, G., Pratt, H., Michalewski, H. J., \& Patterson, J. V. (1990). Electrophysiological measures of short-term memory. In T. Shallice \& G. Vallar (Eds.), Neuropsychological impairments of short-term memory (pp. 94-110). New York: Cambridge University Press.

SzirTes, J., \& VAUghaN, H. G., JR. (1977). Characteristics of cranial and facial potentials associated with speech production. Electroencephalography \& Clinical Neurophysiology, 43, 386-396.

VALLAR, G., \& CAPPA, S. F. (1987). Articulation and verbal short-term memory: Evidence from anarthria. Cognitive Neuropsychology, 4, $55-78$
VAllar, G., CoRno, M., \& Basso, A. (1992). Auditory and visual shortterm memory in aphasia. Cortex, 28, 383-389.

Woods, D. L. (1990). The physiological basis of selective attention: Implications of event-related potential studies. In J. W. Rohrbaugh, R. Parasuraman, \& R. Johnson, Jr. (Eds.), Event-related brain potentials: Basic issues and applications (pp. 179-209). New York: Oxford University Press.

Woods, D. L., AlHo, K., \& AlgaZI, A. (1991). Brain potential signs of feature processing during auditory selective attention. NeuroReport, 2, 189-192.

Woods, D. L., Hillyard, S. A., \& Hansen, J. C. (1984). Event-related brain potentials reveal similar attentional mechanisms during selective listening and shadowing. Journal of Experimental Psychology: Human Perception \& Performance, 10, 761-777.

\section{NOTE}

1. In a recent study, we (Martin-Loeches, Schweinberger, \& Sommer, 1997) have replicated our event-related potential pattern for the auditory phonological similarity effect even in the absence of any irrelevant material. Interestingly, phonological similarity in the visual modality increased an occipital N1 component. Therefore we concluded that the best candidate for our auditory phonological similarity effect is not the phonological N400 - which would be expected to be modality independent-but the Nd component. Both auditory Nd and visual NI enhancement reflect functionally equivalent phenomena (selective attention), but specific for each modality.

(Manuscript received June 24, 1996; revision accepted for publication September 19, 1996.)

\title{
Nominations for the Editorship of Perception \& Psychophysics
}

Nominations are solicited for the editorship of Perception \& Psychophysics. The term of the present editor, Myron Braunstein, expires at the end of 1998. The new editor will begin an official 4- or 5-year term on January 1, 1999, and will begin to receive manuscripts early in January 1998. The Publications Committee of the Psychonomic Society expects to appoint the new editor by November 1997.

Nominations (including self-nominations) should be submitted by August 1, 1997, to:

\author{
Stephen Palmer \\ Chair, Perception \& Psychophysics Search Committee \\ Department of Psychology \\ University of California \\ Berkeley, CA 94720-1650
}

